\title{
Oxytocin Receptor Gene Methylation as a Molecular Marker for Severity of Depressive Symptoms in Affective Disorder Patients
}

\section{Birgit Ludwig}

Medical University of Vienna

Laura Carlberg

Medical University of Vienna

Klemens Kienesberger

Medical University of Vienna

Patrick Swoboda

Medical University of Vienna

Marleen Swoboda

Universitätsklinikum Tulln

Alexandra Bernegger

Hospital of the Brothers of St. John of God

Romina Koller

Medical University of Vienna

Michelle Inaner

Medical University of Vienna

Monika Fuxjäger

Medical University of Vienna

Melanie Zotter

Zentrum für Seelische Gesundheit

Nicolas Schmelzle

Medical University of Vienna

Birgit Senft

Zentrum für Seelische Gesundheit

Lisa Meisner

Zentrum für Seelische Gesundheit

Daniela Fischer-Hansal

Zentrum für Seelische Gesundheit

Jasmin Huber

Austrian Institute of Technology

Silvia Schönthaler 
Austrian Institute of Technology

Nestor D. Kapusta

Medical University of Vienna

Helmuth Haslacher

Medical University of Vienna

Martin Aigner

Universitätsklinikum Tulln

Andreas Weinhaeusel

Austrian Institute of Technology

Siegfried Kasper

Medical University of Vienna

Alexandra Schosser ( $\nabla$ alexandra.schosser@mail.sfu.ac.at )

Sigmund Freud University Vienna

\section{Research Article}

Keywords: Oxytocin Receptor Gene, methylation, major depression, bipolar disorder, depressive episode, childhood trauma questionnaire

Posted Date: December 6th, 2021

DOI: https://doi.org/10.21203/rs.3.rs-1115325/v1

License: (c) (1) This work is licensed under a Creative Commons Attribution 4.0 International License.

Read Full License 


\section{Abstract}

Background: Oxytocin (OXT) is a neuropeptide and hormone involved in emotional functioning and also seems to play a role in moderating the stress response. Both preclinical and clinical studies point to an increased methylation status of the Oxytocin receptor (OXTR) promoter region with concomitant deficits in social, cognitive and emotional functioning. We hypothesize that methylation levels (\%) of the oxytocin receptor promoter region correlate with the severity of depression symptoms within this present sample of affective disorder patients.

Methodology: Eight hundred forty six Caucasian affective disorder patients were recruited the Department of Psychiatry and Psychotherapy of the Medical University Vienna, the Karl Landsteiner University for Health and Science and Zentrum für seelische Gesundheit, BBRZ-Med Leopoldau. The assessment included an assemblage of psychiatric interviews (e.g. SCAN, HAMD, CTQ) and concomitantly DNA samples of peripheral blood cells were collected for Multiplexed and Sensitive DNA Methylation Testing.

Results: Our data suggests a positive but not significant association between OXTR promoter Exons 1-3 methylation levels and severity of depression symptoms in affective disorder patients.

Conclusions: Our findings contribute to elucidate the role of OXTR in affective disorders, but further longitudinal studies in particular are necessary to broaden the current state of knowledge.

\section{Background}

Oxytocin (OXT) is a neuropeptide and hormone involved in emotional functioning and also seems to play a role in moderating the stress response. Oxytocin receptors are synthesized by the Oxytocin Receptor (OXTR) gene and expressed both in the brain and peripheral organs. The OXTR gene spans 17 kilobytes $(\mathrm{kb})$ and contains 4 exons and 3 introns. While exons 1 and 2 correspond to non-coding regions, exons 3 and 4 encode the amino acids of the OXTR gene. Molecular-wise it is a class I G-protein coupled transmembrane receptor, facilitating the oxytocin pathway [1].

One of the most established epigenetic mechanisms is DNA methylation. High levels of DNA methylation are commonly known to decrease mRNA expression, differential methylation is one of the known mechanisms to regulate gene expression [2]. The functional significance of OXTR DNA methylation has been supported by both rodent and clinical data. Researchers from the University of Haifa showed that the mouse oxytocin receptor gene was epigenetically regulated by DNA methylation of its promoter and they also found that specific $\mathrm{CpG}$ sites were differentially methylated between distinct murine brain regions expressing different levels of OXTR mRNA $[3,4]$.

Findings in psychiatric disorders in general point to an increased methylation status of the OXTR promoter region with decreased peripheral gene expression in individuals afflicted with depression, anxiety or situations of increased stress $[5,6]$. 
Preclinical findings suggest that long-term isolation down-regulates OXTR mRNA transcription and may contribute to the development of depression in isolated mice through the intracentral amygdala injection of OXT [7]. In a rodent model of postpartal depression, using a social stress paradigma during lactation, a significant decrease in oxytocin mRNA expression in the medial amygdala was found [8]. More recent preclinical findings in female rodents suggest that chronic social defeat stress might increase the levels of anxiety and depression via a reduction in oxytocin projections and the oxytocin receptor level in the nucleus accumbens [9]. Clinical data suggests that mothers with early trauma (loss of a parent or sexual abuse in childhood) had lower serum OXTR mRNA than no-trauma mothers [10]. There is evidence suggesting that both Major Depression Disorder (MDD) and Bipolar Disorder (BPD) patients show increased expression of OXTR mRNA in the dorsolateral prefrontal cortex compared to patients with schizophrenia and controls [11]. OXTR protein expression levels were significantly decreased in women having suffered from exposure to childhood trauma, and there was a also a significant negative correlation between OXTR protein expression levels and the CTQ score [12].

Previous publications report inconclusive results regarding the implication of OXTR

promoter methylation in affective disorders but point in general to an increase of OXTR gene promoter methylation in depressed individuals $[5,6]$.

Rainer et al. [13] reported significantly decreased methylation of Exon I in the oxytocin receptor promoter region in depressed female vs. healthy female controls [13]. A similar study investigating postmenopausal women with anxiety and depression, found oxytocin receptor methylation to be increased in a subgroup of rs53576 AA carriers when compared to healthy age-matched female controls [14]. Another study focusing on postpartal depressive women found increased DNA methylation of the OXTR only in individuals with a rs53576 GG genotype [15].

A Japanese study focusing on children with and without a history of childhood maltreatment compared salivary OXTR methylation levels which resulted in higher $\operatorname{CpG~5,6~methylation~of~the~maltreated~children~}$ [16]. Smearman et al. [17] attempted to unveil the associations between early life adversities, DNA methylation of OXTR gene and adult psychiatric symptoms. Child abuse was associated with higher methylation of two $\mathrm{CpG}$ sites, yet did not survive correction for multiple testing nor served as a mediator of adult psychopathology [17].

In a study including 309 African American men, childhood adversity was not directly associated with elevated OXTR methylation but could be explained by the factor of socioeconomic precarity [18].

The OXTR and its methylation status might be associated with early life adversity and the pathogenesis of MDD during life time. In an attempt to refine the biopsychosocial model of the pathogenesis of affective disorders, we aimed to explore if the OXTR methylation status was associated with depressive symptoms and/or a history of childhood maltreatment in a large cohort of affective disorder patients.

\section{Methods}




\section{- Participants}

A total of 846 unrelated in- and outpatients with affective disorders were recruited at three study sites in Vienna and the surrounding area. A total of 382 patients were recruited at the Department of Psychiatry and Psychotherapy of the Medical University Vienna, 67 patients Karl Landsteiner University for Health and Science and 397 patients at the Zentrum für seelische Gesundheit, BBRZ-Med Leopoldau in the context of the Austrian Science Funds (FWF) funded study "VieSAD" ("Vienna Study on Genetics of Suicidal Behavior in Affective Disorders", KLI'220). The investigation was carried out in accordance with the latest version of the Declaration of Helsinki and approval for the study was obtained from the Ethical Committee of the Medical University of Vienna (EK 2013/2013) and the federal state of Lower Austria (GS4- EK-4/181/2012).

Caucasians aged from 18 to 65 years were included if they were diagnosed with either bipolar disorder (BD) or major depressive disorder (MDD) as defined by ICD-10 and/or DSM-IV criteria. Exclusion criteria were mood incongruent psychotic symptoms or lifetime history of schizophrenia, primary organic disease, primary substance abuse, pregnancy and breastfeeding. Diagnosis was affirmed by performing detailed clinical examination (SCAN- Schedules for Clinical Assessment in Neuropsychiatry[19]). In order to screen for acute affective states, the HAMD (Hamilton Depression Scale [23]) was applied when blood for genotyping was drawn. Comorbidities were monitored, as well as weight, height and Body Mass Index. In a face-to-face interview, patients were informed about the study and signed a written consent form. Interrater reliability was guaranteed by extensive interview training, following Good Clinical Practice criteria. Biomaterial was processed and stored at the MedUni Wien Biobank facility in an ISO 9001certified environment according to standard operating procedures published previously (Haslacher et al., 2018).

\section{- Statistical analyses}

All statistics were conducted using the statistical software SPSS 22.0 (IBM, Armonk USA) and 'R 3.4.2' (cran.r-project.org/). Continuous data were presented as mean and standard deviation, respectively with confidence intervals. Categorical data were given as counts and percentages. Normal distribution of the variables was tested by Shapiro-Wilk test. None of the tested variables were normally distributed (OXTR methylation: $W=0.129, p<0.001, n=748$; HAMD score: $W=0.994, p=0.002, n=748$; CTQ score: $W=0.921$, $\mathrm{p}<0.001, \mathrm{n}=748$ ). Consecutively, non-parametric testing was applied for all variables. Differences between more than two groups were assessed by means of the Kruskall-Wallis H Test. For non-parametric correlation data, Kendall-Tau-b correlation coefficient was calculated. All test results were interpreted twotailed with a significance level established at $p \leq 0.05$.

Power analyses were performed using the software R (Version 1.1.456) with the " $R$ " package "pwr". The appropriate sample size was estimated along the lines of Cohen (1988) (pwr.t.test $(n=N U L L, d=0.5$, sig.level $=0.05$, power $=0.80$, type $=c($ "two.sample"), alternative $=c($ "two.sided")). Assuming a medium clinical effect size of $d=0.5$, one sample, and accepting an a-error of 0.05 and a $\beta$-error of 0.2 (power=0.8), those effects would be detectable at a sample size $\geq 34$ per group. For ANOVA comparing 5 samples, 
assuming a medium effect of $f=0.4$, accepting an a-error of 0.05 and a $\beta$-error of 0.2 (power=0.8), those effects would be detectable at a sample size $\geq 16$ per group.

\section{- Methylation analysis}

Epigenetic methylation analyses were performed using the MSRE (methylation-sensitive restriction enzyme) - qPCR (quantitative polymerase chain reaction) approach [26]. For methylation analyses, based on previous literature, the CpG covering part of OXTR Exons 1-3 promoter region (chr3:8,769,499$8,769,620$,hg 38) with a size of 121 bp (PCR primers - forward: gctggggctgaggctgcactatc and reverse: cccatttgttaaggctctgggaccaa), containing a total of $12 \mathrm{CpG}$ sites; of those $6 \mathrm{CpGs}$ were covered by the methylation sensitive restriction enzymes Acil 32, Acil 42, Acil 56, Hin6i 58, Hpall 70, and Acil 75. An additional figure file shows this in more detail [see Additional file 1].

Digestion of all samples and parallel incubation without digestion were performed the same day. $2 \mu \mathrm{l}$ digested/undigested DNA was used for the following qPCR protocol: hotstart activation with $95^{\circ} \mathrm{C}$ for $5 \mathrm{~min}$, followed by amplification (45x) with $95^{\circ} \mathrm{C}$ for $40 \mathrm{sec}, 65^{\circ} \mathrm{C}$ for $40 \mathrm{sec}, 72^{\circ} \mathrm{C}$ for $1 \mathrm{~min} 20 \mathrm{sec}$, followed by the final extension step with $72^{\circ} \mathrm{C}$ for $7 \mathrm{~min}$ and cool-down phase with $4^{\circ} \mathrm{C}$. Raw data of methylation analyses (Ct- and Tm-values) were calculated. Methylation status in \% was calculated relatively to the reference values of undigested DNA samples applying the following formula:

$\%$ methylation $=$ sample digested $[\mathrm{ng}] /$ sample undigested $[\mathrm{ng}] * 100 \%$

The according values were reported as percentage of methylated reference (PMR) values.

\section{Results}

In total, 846 patients were recruited. Methylation data was available for a total of 814 patients. Both HAMD data and methylation data were available for a total of 757 patients. Both complete CTQ data and methylation data were available for 751 patients. HAMD, CTQ and methylation data were available for a total of 748 patients, these are further analyzed in the following section.

The mean age of the participants was 44.94 years $(S D= \pm 12.9, n=748), 489$ of them were women $(65,4 \%)$ and 259 of them were men $(34.6 \%)$. A total of 656 patients were included with a diagnosis of Major Depression Disorder, a total of 92 patients were included with a diagnosis of Bipolar Disorder.

\section{- Methylation analysis}

To examine the methylation status of OXTR gene promoter region, the MSRE - qPCR approach was performed. Since there was no significant difference between male and female affective disorder patients in the PMR values of the OXTR gene in the present sample $(f=0.29 \pm 0.03, m=0.45 \pm 0.16 ; U=0.461 ; p=$ $0.65, n=748$, Mann-Whitney-U-test), all further analyses were exercised for the entire sample. Since there was no significant correlation between age and the PMR values of the OXTR gene as inferred from the 
Kendall-Tau-b correlation coefficient $(\tau=0.032, p=0.20, n=748)$, further analyses were not corrected for age.

One-way ANOVA with Kruskall-Wallis was applied on HAMD score categories, differentiating between no symptoms $(n=78)$, mild symptoms $(n=191)$, moderate symptoms $(n=249)$, severe symptoms $(n=121)$ and very severe depressive symptoms $(n=116)$. The analysis resulted in no significant differences in between categories based on the PMR values of OXTR gene promotor methylation ( $W=6.544 ; p=0.16, n=748)$ (Figure 1).

A one-way ANOVA with Kruskall-Wallis comparing oxytocin gene promoter methylation (\%) between none to low trauma exposure, low to moderate trauma exposure, moderate to severe trauma exposure and severe to extreme trauma exposure within the CTQ subscales showed no significant differences in any of the subscales (Table 1).

Table 1

CTQ subscales with classification according to Bernstein comparing mean

\begin{tabular}{|llll|}
\hline CTQ-Subscale & $\boldsymbol{H}$ & $\mathbf{p}$-value & $\mathbf{n}$ \\
\hline Emotional Abuse & 4.144 & 0.25 & 748 \\
\hline Emotional Neglect & 4.507 & 0.21 & 748 \\
\hline Physical Abuse & 5.028 & 0.17 & 748 \\
\hline Physical Neglect & 0.553 & 0.91 & 748 \\
\hline Sexual Abuse & 5.324 & 0.15 & 748 \\
\hline
\end{tabular}

PMR values of the OXTR promotor region (Oneway-ANOVA Kruskall-Wallis with pair-wise comparison)

An additional figure file shows this in more detail [see Additional file 2]

In this study investigating affective disorder patients, there was also a highly significant positive correlation ( $\tau=0.15, p<0.0001, n=788$ ) between the total Hamilton score and the total CTQ-score as we already reported previously [27]. An additional figure file shows this in more detail [see Additional file 3].

\section{Discussion}

The aim of the current study was to investigate a possible association between the methylation status of the OXTR gene promoter region and severity of depression symptoms as well as early life adversities.

Several publications suggest sex differences in the methylation status of OXTR promoter region [28, 29], but in our cohort (748 analyzed samples) these findings could not be replicated. Regarding the 
comparison with Nawjin et al's study it must be noted, that they found sex-specific methylation patterns comparing female and male Posttraumatic Stress Disorder (PTSD) patients (total of 62 samples) in OXTR exon 3. Our study included a total of 808 analyzed samples of the exon 1 OXTR gene promoter region in diagnosed affective disorder patients: no significantly different PMR values were found between affected men and women. The second referenced study also has important differences with our study: their patients were diagnosed with psychotic disorders (total of 242) and again a different region within the OXTR promotor was analyzed [29]. Recent research suggests that females might be more sensitive to the impact of early life adversities on OXTR methylation [30]. In the same study they also found sex differences in a cohort of men and women without any previous diagnosis. Women had significantly lower DNA methylation in the promoter region than males, but higher DNA methylation in two analyzed Intron regions [30]. Although sex differences were not significant in our sample, we also found women to have lower DNA methylation in the promoter region of OXTR gene than men.

Our main interest was to determine if the OXTR promoter region methylation status was associated with the severity of depressive symptoms in affective disorder patients. Using an established depression questionnaire at the time of blood draw, we aimed to correlate biological markers with clinical parameters from standardized questionnaires in a timely manner. The present results suggest that severity of depression in affective disorders is not significantly associated with PMR values of OXTR promoter region. To the best of our knowledge, this is study is the first one to examine the severity of depressive symptoms in relation to the methylation status of OXTR promoter region.

Another interest of the present study was to determine if the methylation status of OXTR promoter region was associated with early life adversities. Again, a standardized questionnaire (CTQ) was used to establish if and to what extent these affective disorder patients had experienced childhood trauma. Overall, there was no significant association between the CTQ score subscales and PMR values.

$\mathrm{CpG}$ islands tend to be unmethylated and higher PMR values of methylation in $\mathrm{CpG}$ islands within gene promoter regions tend to correlate with a repression of gene (and protein) expression in the corresponding tissue. The already mentioned studies by Krause et al. [12] and Light et al. [10] showed a negative association between OXTR protein/gene expression and CTQ-score, which aligns with the trend in our findings.

Limitations of this study include, that $\mathrm{CpG}$ islands were not specified but the methylation of $6 \mathrm{CpGs}$ was analyzed as a whole. More specific analyses as done by Fujisawa et al. [16] might have resulted in more precise results. Another limitation is the retrospective analysis of data. We included and interviewed all affective disorder patients meeting the inclusion criteria, not based on their current severity of depression. Consequently, when comparing the groups of different severity of depression, the individual group size differs, but in total the divided group sizes were still bigger than the total of included samples in most of the studies referenced in this manuscript.

One of the clear strengths of this study is the precise phenotypic definition of our sample, which results in a highly homogeneous sample (namely affective disorder patients only) and allows us to prevent 
interference of the confounding affective disorders phenotype. Another strength of this study is the large sample size of 748 analyzed samples of affective disorder patients, compared to previous clinical methylation studies.

\section{Conclusions}

In conclusion, the herein presented results could not support the hypothesis that increased methylation of OXTR gene promoter region and supposedly downregulation of OXTR in peripheral tissues is associated with a higher severity of depressive symptoms in affective disorder patients. Neither could we show a significant association between higher methylation levels of OXTR gene promoter region and higher CTQ scores within the subscale. But as shown before, early life adversities and depressive symptoms significantly correlate within our sample [27].

In this study we attempted to elucidate the role of OXTR methylation in affective disorders, further studies

Our findings contribute to elucidate the role of OXTR in affective disorders, but further longitudinal studies in particular are necessary to broaden the current state of knowledge.

\section{Abbreviations}

BPD

Bipolar Affective Disorder

CTQ

Childhood Trauma Questionnaire

HAMD

17-item Hamilton Rating Scale for Depression

MDD

Major Depressive Disorder

MSRE

Methylation-sensitive restriction enzyme

OR

Odds Ratio

OXT

Oxytocin

OXTR

Oxytocin Receptor

PMR

Percentage of Methylation Ratio

PTSD

Posttraumatic Stress Disorder

SCAN

Schedules for Clinical Assessment in Neuropsychiatry

VieSAD

Vienna Study on Genetics of Suicidal Behavior in Affective Disorders

\section{Declarations}




\section{Ethics approval and consent to participate}

The investigation was carried out in accordance with the latest version of the Declaration of Helsinki and approval for the study was obtained from the Ethical Committee of the Medical University of Vienna (EK 2013/2013) and the federal state of Lower Austria (GS4- EK-4/181/2012). Informed consent was obtained from all participants.

\section{Consent for publication}

Not applicable.

\section{Availability of data and materials}

The datasets supporting the conclusions of this article are available in the repository [https://mfr.osf.io/render?url=https\%3A\%2F\%2Fosf.io\%2Fqwmkj\%2Fdownload].

\section{Competing interests}

The authors Ludwig, Carlberg, Kienesberger, Swoboda P, Swoboda M, Bernegger, Koller, Inaner, Senft, Meisner, Fuxjäger, Zotter, Fischer-Hansal, Schmelzle, Huber, Schoenthaler, Kapusta, Haslacher, Weinhaeusel, Aigner, and Schosser declare no competing interests. Siegfried Kasper received grants/research support, consulting fees and/or honoraria within the last three years from Angelini, AOP Orphan Pharmaceuticals AG, Celegne GmbH, Eli Lilly, Janssen-Cilag Pharma GmbH, KRKA-Pharma, Lundbeck A/S, Mundipharma, Neuraxpharm, Pfizer, Sanofi, Schwabe, Servier, Shire, Sumitomo Dainippon Pharma Co. Ltd. and Takeda.

\section{Funding}

This study was funded by a research grant (KLI²20) for "VieSAD" ("Vienna Study on Genetics of Suicidal Behavior in Affective Disorders") from the Austrian Science Funds (FWF).

\section{Authors' contributions}

BL: Conception and design, acquisition of data, analysis and interpretation of data, manuscript writing, final approval of the version to be published

LC: Acquisition of data, analysis and interpretation of data, manuscript revision, final approval of the version to be published 
KK: Acquisition of data, analysis and interpretation of data, manuscript writing, final approval of the version to be published

PS: Acquisition of data, manuscript revision, final approval of the version to be published MS: Acquisition of data, manuscript revision, final approval of the version to be published $A B$ : Acquisition of data, manuscript revision, final approval of the version to be published RK: Acquisition of data, manuscript revision, final approval of the version to be published Ml: Acquisition of data, manuscript revision, final approval of the version to be published MF: Acquisition of data, manuscript revision, final approval of the version to be published MZ: Acquisition of data, manuscript revision, final approval of the version to be published NS: Acquisition of data, manuscript revision, final approval of the version to be published BS: Acquisition of data, manuscript revision, final approval of the version to be published LM: Acquisition of data, manuscript revision, final approval of the version to be published DHF: Interpretation of data, manuscript revision, final approval of the version to be published $\mathrm{JH}$ : Acquisition of data, manuscript revision, final approval of the version to be published SS: Acquisition of data, manuscript revision, final approval of the version to be published NDK: Conception and design, acquisition of data, analysis and interpretation of data, manuscript revision, final approval of the version to be published

$\mathrm{HH}$ : Analysis and interpretation of data, manuscript writing, final approval of the version to be published MA: Conception and design, acquisition of data, analysis and interpretation of data, manuscript revision, final approval of the version to be published

AW: Analysis and interpretation of data, Acquisition of data, manuscript revision, final approval of the version to be published

SK: Conception and design, acquisition of data, analysis and interpretation of data, manuscript revision, final approval of the version to be published

AS: Principal investigator of the VieSAD study, conception and design, acquisition of data, analysis and interpretation of data, manuscript revision, final approval of the version to be published

All authors contributed to, read and approved the final manuscript. 


\section{Acknowledgements}

Not applicable.

\section{References}

1. Gimpl G, Fahrenholz F: The oxytocin receptor system: structure, function, and regulation. Physiol Rev 2001, 81(2):629-683.

2. Siegfried Z, Eden S, Mendelsohn M, Feng X, Tsuberi BZ, Cedar H: DNA methylation represses transcription in vivo. Nat Genet 1999, 22(2):203-206.

3. Harony-Nicolas H, Mamrut S, Brodsky L, Shahar-Gold H, Barki-Harrington L, Wagner S: Brain regionspecific methylation in the promoter of the murine oxytocin receptor gene is involved in its expression regulation. Psychoneuroendocrinology 2014, 39:121-131.

4. Mamrut S, Harony H, Sood R, Shahar-Gold H, Gainer H, Shi YJ, Barki-Harrington L, Wagner S: DNA methylation of specific $\mathrm{CpG}$ sites in the promoter region regulates the transcription of the mouse oxytocin receptor. PLoS One 2013, 8(2):e56869.

5. Maud C, Ryan J, Mclntosh JE, Olsson CA: The role of oxytocin receptor gene (OXTR) DNA methylation (DNAm) in human social and emotional functioning: a systematic narrative review. $B M C$ Psychiatry 2018, 18(1):154.

6. Chen D, Meng L, Pei F, Zheng Y, Leng J: A review of DNA methylation in depression. J Clin Neurosci 2017, 43:39-46.

7. Han RT, Kim YB, Park EH, Kim JY, Ryu C, Kim HY, Lee J, Pahk K, Shanyu C, Kim H et al: Long-Term Isolation Elicits Depression and Anxiety-Related Behaviors by Reducing Oxytocin-Induced GABAergic Transmission in Central Amygdala. Front Mol Neurosci 2018, 11:246.

8. Murgatroyd CA, Taliefar M, Bradburn S, Carini LM, Babb JA, Nephew BC: Social stress during lactation, depressed maternal care, and neuropeptidergic gene expression. Behav Pharmacol 2015, 26(7 Spec No):642-653.

9. Hou W, He Z, Yang Y, Yuan W, Wang L, Zhang J, Zhang X, Cai W, Guo Q, Tai F: The involvement of oxytocin in the effects of chronic social defeat stress on emotional behaviours in adult female mandarin voles. Eur J Neurosci 2020, 52(2):2853-2872.

10. Light AE, Holt-Lunstad J, Porter CL, Light KC: Early life trauma: An exploratory study of effects on OXTR and NR3C1 gene expression and nurturing self-efficacy in mothers of infants. Int $J$ Psychophysiol 2019, 136:64-72.

11. Lee MR, Sheskier MB, Farokhnia M, Feng N, Marenco S, Lipska BK, Leggio L: Oxytocin receptor mRNA expression in dorsolateral prefrontal cortex in major psychiatric disorders: A human post-mortem study. Psychoneuroendocrinology 2018, 96:143-147.

12. Krause S, Boeck C, Gumpp AM, Rottler E, Schury K, Karabatsiakis A, Buchheim A, Gundel H, Kolassa IT, Waller C: Child Maltreatment Is Associated with a Reduction of the Oxytocin Receptor in Peripheral 
Blood Mononuclear Cells. Front Psychol 2018, 9:173.

13. Reiner I, Van IMH, Bakermans-Kranenburg MJ, Bleich S, Beutel M, Frieling H: Methylation of the oxytocin receptor gene in clinically depressed patients compared to controls: The role of OXTR rs53576 genotype. J Psychiatr Res 2015, 65:9-15.

14. Chagnon YC, Potvin O, Hudon C, Preville M: DNA methylation and single nucleotide variants in the brain-derived neurotrophic factor (BDNF) and oxytocin receptor (OXTR) genes are associated with anxiety/depression in older women. Front Genet 2015, 6:230.

15. Bell AF, Carter CS, Steer CD, Golding J, Davis JM, Steffen AD, Rubin LH, Lillard TS, Gregory SP, Harris JC et al: Interaction between oxytocin receptor DNA methylation and genotype is associated with risk of postpartum depression in women without depression in pregnancy. Front Genet 2015, 6:243.

16. Fujisawa TX, Nishitani S, Takiguchi S, Shimada K, Smith AK, Tomoda A: Oxytocin receptor DNA methylation and alterations of brain volumes in maltreated children. Neuropsychopharmacology 2019, 44(12):2045-2053.

17. Smearman EL, Almli LM, Conneely KN, Brody GH, Sales JM, Bradley B, Ressler KJ, Smith AK: Oxytocin Receptor Genetic and Epigenetic Variations: Association With Child Abuse and Adult Psychiatric Symptoms. Child Dev 2016, 87(1):122-134.

18. Kogan SM, Bae D, Cho J, Smith AK, Nishitani S: Childhood Adversity, Socioeconomic Instability, Oxytocin-Receptor-Gene Methylation, and Romantic-Relationship Support Among Young African American Men. Psychol Sci 2019, 30(8):1234-1244.

19. Wing JK, Babor T, Brugha T, Burke J, Cooper JE, Giel R, Jablenski A, Regier D, Sartorius N: SCAN. Schedules for Clinical Assessment in Neuropsychiatry. Archives of general psychiatry 1990, 47(6):589-593.

20. Kapusta N: P03-451 - Develpment of a suicide risk assessment scale - surias. Eur Psychiatry 2011, 32(Suppl1):1621.

21. Osman A, Bagge CL, Gutierrez PM, Konick LC, Kopper BA, Barrios FX: The Suicidal Behaviors Questionnaire-Revised (SBQ-R): validation with clinical and nonclinical samples. Assessment 2001, 8(4):443-454.

22. Linehann MC, K: Lifetime Parasuicide Count (LPC). Seattle:University of Washington 1994.

23. Hamilton M: Development of a rating scale for primary depressive illness. The British journal of social and clinical psychology 1967, 6(4):278-296.

24. Montgomery SA, Asberg M: A new depression scale designed to be sensitive to change. The British journal of psychiatry: the journal of mental science 1979, 134:382-389.

25. Asberg M, Traskman L, Thoren P: 5-HIAA in the cerebrospinal fluid. A biochemical suicide predictor? Archives of general psychiatry 1976, 33(10):1193-1197.

26. Beikircher G, Pulverer W, Hofner M, Noehammer C, Weinhaeusel A: Multiplexed and Sensitive DNA Methylation Testing Using Methylation-Sensitive Restriction Enzymes "MSRE-qPCR". Methods Mol Biol 2018, 1708:407-424. 
27. Bernegger A, Kienesberger K, Carlberg L, Swoboda P, Ludwig B, Koller R, Kapusta ND, Aigner M, Haslacher $\mathrm{H}$, Schmoger $\mathrm{M}$ et al: Influence of Sex on Suicidal Phenotypes in Affective Disorder Patients with Traumatic Childhood Experiences. PLoS One 2015, 10(9):e0137763.

28. Nawijn L, Krzyzewska IM, van Zuiden M, Henneman P, Koch SBJ, Mul AN, Frijling JL, Veltman DJ, Mannens $\mathrm{M}$, Olff $\mathrm{M}$ : Oxytocin receptor gene methylation in male and female PTSD patients and trauma-exposed controls. Eur Neuropsychopharmaco/2019, 29(1):147-155.

29. Rubin LH, Connelly JJ, Reilly JL, Carter CS, Drogos LL, Pournajafi-Nazarloo H, Ruocco AC, Keedy SK, Matthew I, Tandon $\mathrm{N}$ et al: Sex and diagnosis specific associations between DNA methylation of the oxytocin receptor gene with emotion processing and temporal-limbic and prefrontal brain volumes in psychotic disorders. Biol Psychiatry Cogn Neurosci Neuroimaging 2016, 1(2):141-151.

30. Gouin JP, Zhou QQ, Booij L, Boivin M, Cote SM, Hebert M, Ouellet-Morin I, Szyf M, Tremblay RE, Turecki $\mathrm{G}$ et al: Associations among oxytocin receptor gene (OXTR) DNA methylation in adulthood, exposure to early life adversity, and childhood trajectories of anxiousness. Sci Rep 2017, 7(1):7446.

\section{Figures}

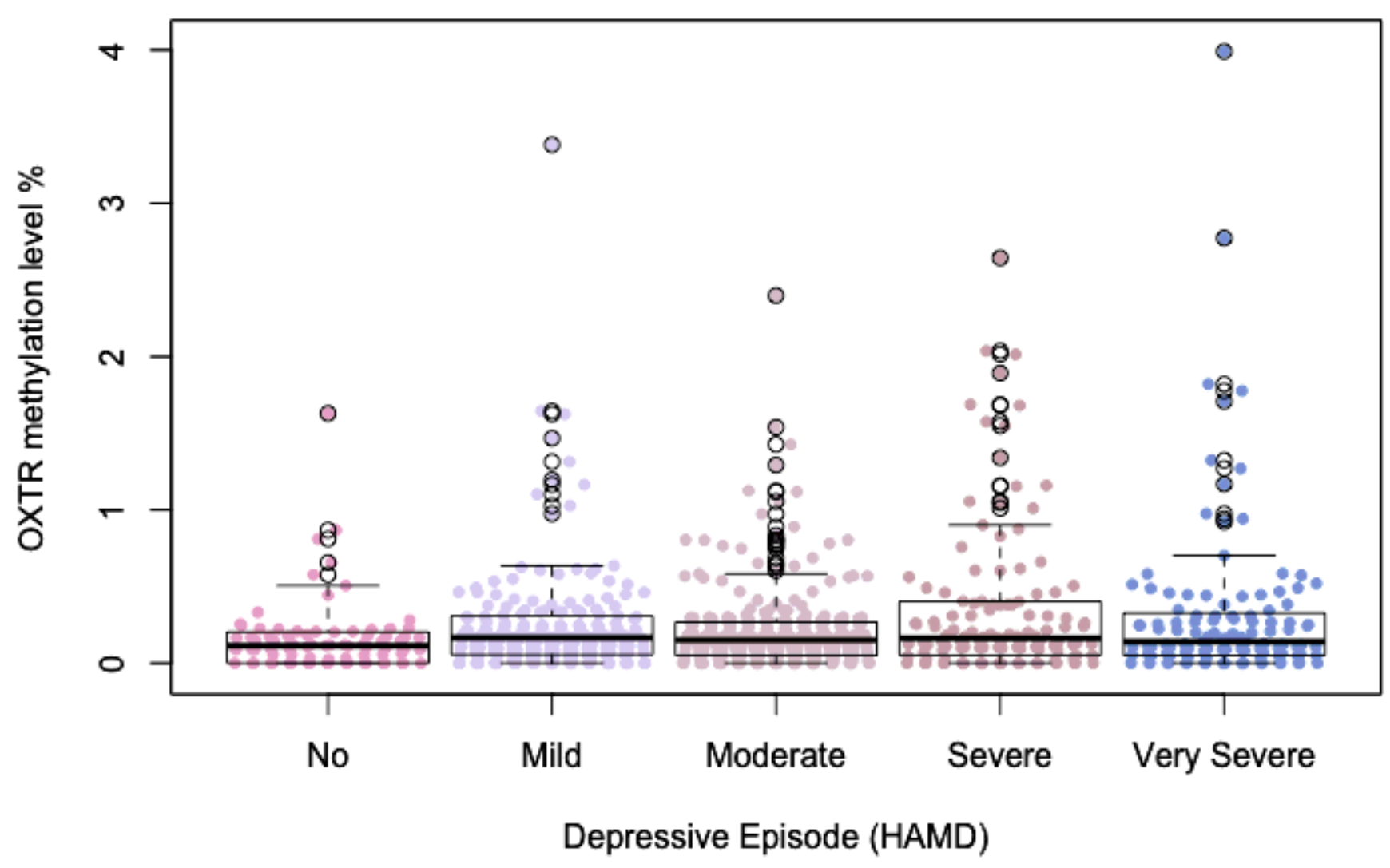

Figure 1 
Box plots presenting HAMD score categories, differentiating between no symptoms ( $n=78)$, mild symptoms $(n=191)$, moderate symptoms $(n=249)$, severe symptoms $(n=121)$ and very severe depressive symptoms $(n=116)$ based on the percentage of methylation ratio (PMR) values of OXTR gene promotor methylation. The analysis resulted in no significant differences in between categories based on the PMR values of OXTR gene promotor methylation $(W=6.544 ; p=0.16, n=748)$. Outliers (detected with Ztransformation) were removed from the graph for a better visualization of the results (whereas within the statistical analyses no outliers were removed).

\section{Supplementary Files}

This is a list of supplementary files associated with this preprint. Click to download.

- AdditionalFile1.pdf

- AdditionalFile2.pdf

- AdditionalFile3.pdf 